\title{
Magnetic Structure Equilibria and Evolutions due to Active Region Interactions
}

\author{
J. Lin and A. A. van Ballegooijen \\ Harvard-Smithsonian Center for Astrophysics, 60 Garden Street, Cambridge, MA 02138, USA \\ email: jlin@cfa.harvard.edu,vanballe@cfa.harvard.edu
}

\begin{abstract}
Equilibria and evolutions in the coronal magnetic configurations due to the interactions among active regions are investigated. The magnetic structure includes a current-carrying flux rope that is used to model the prominence or filament. We use either two dipoles or four monopoles on the boundary surface to model active regions, and the change in the boundary conditions corresponds to either the horizontal motion of magnetic sources or decaying of the active regions. Both cases show the catastrophic behavior in the system's evolutions. The results have important observational consequences: most eruptive prominences that give rise to CMEs are driven by the interactions between two or more active regions. Such eruptions may not necessarily take place in the growing phase of the active regions, instead they usually occur at the decay phase.
\end{abstract}

Keywords. Sun: coronal mass ejections (CMEs), evolution, filaments, MHD

\section{Introduction}

In response to slow motions in the photosphere and in the convective zone, the coronal magnetic field evolves and builds up the stress and the extra energy. In addition to the usual forms of motions, such as shearing and converging of the footpoints of the magnetic field, and the new emerging flux, the system's energy can also increase due to the interaction among two or more active regions as they approach one another. In observations, the horizontal motions of active regions (or sunspots) have long been a well-known precursor for some types of eruptions (Tanaka \& Nakagawa 1973; Martres et al. 1986; Dezsö et al. 1984; Kovács \& Dezsö 1986; Dezsö \& Kovács 1998).

Previously, we investigated the detailed consequences of the new emerging flux, and discussed the results of the horizontal motions of the magnetic source regions on the boundary surface (Lin et al. 2001). In the present work, we consider the equilibria and evolutions in the magnetic configurations with background fields resulted from two dipoles or four monopoles located on the boundary surface. All the configurations include a current-carrying flux rope that is usually used to model the prominence or filament. The system evolves as a result of either changes in the strength of the background field, which is utilized to model the magnetic cancellation occurring below and on the surface, or displacement of the source regions on the surface. As we usually do, we are not fuzzy with the details of how a prominence forms. The interested readers may refer to the works by van Ballegooijen \& Martens (1989), van Ballegooijen et al. (2000), as well as MacKay \& van Ballegooijen (2001 and 2005).

\section{Description of Models and Equilibria in Systems}

The model consists of equilibrium solution of the two-dimensional, ideal-MHD equations in the semi-infinite $x-y$ plane $(y \geqslant 0)$ with $y=0$ being the photospheric boundary 


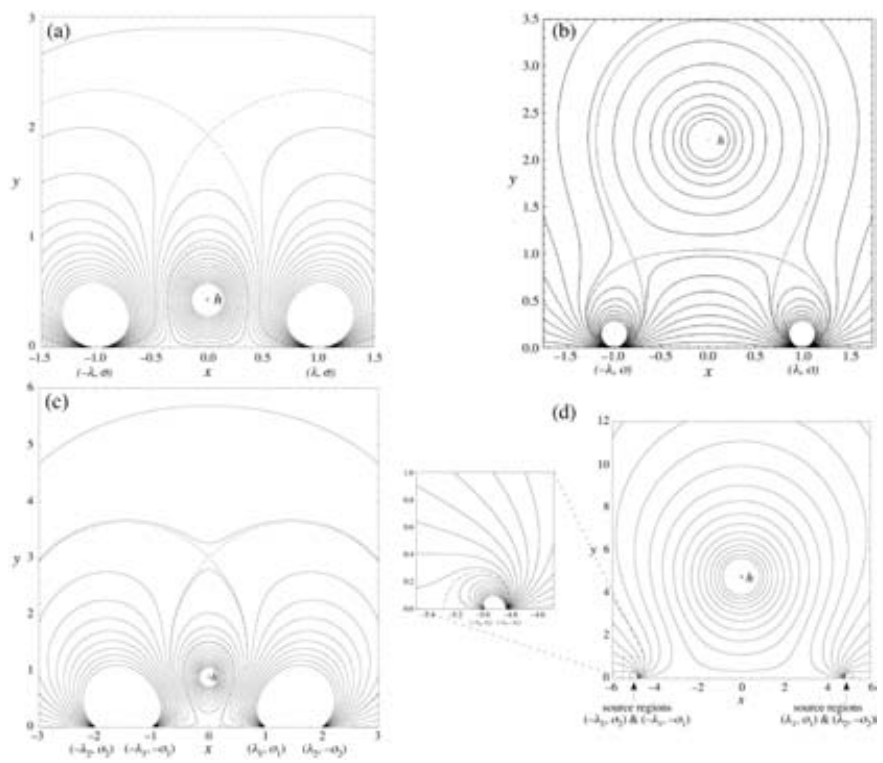

Figure 1. Magnetic configurations with the boundary condition of two dipoles located on the surface: (a) $\lambda=1, \sigma=-2.02$, and $h=0.43$, (b) for $\lambda=1, \sigma=1$, and $h=2.12$; and magnetic configurations with the boundary condition of four monopoles located on the surface: (c) for $\lambda_{1}=1, \lambda_{2}=2, \sigma_{1}=1, \sigma_{2}=1$, and $h=0.89$; and (d) for $\lambda_{1}=4.84, \lambda_{2}=5, \sigma_{1}=1.46, \sigma_{2}=1$, and $h=4.75$. Dashed curves in all the panels plot the separatrices and the magnetic interfaces.

(or more properly, the base of the corona) and $y>0$ corresponding to the corona which is perfectly conducting. So, magnetic field lines in the corona are frozen to the plasma and any reconnection which occurs is restricted to the region where a neutral point or the current sheet exists, and to the photosphere.

In the environment of the low corona, the magnetic field can be treated as force-free and its corresponding evolution prior to the eruption is considered ideal-MHD. We also assume that all the currents are confined in the flux rope and the photosphere, and that there is no current sheet present before the catastrophe takes place (e.g., see Lin et al. 2001; and Lin \& van Ballegooijen 2002). The magnetic configurations of interest are given in Figures 1a and $1 \mathrm{~b}$ for the case in which the background field is produced by two dipoles on the boundary surface; and in Figures 1c and 1d for the case in which the background field results from four monopoles. In each of these panels, $h$ is the height of the flux rope, $\sigma$ is the strength of each dipole, $\lambda$ is the distance between each dipole and the origin, $\pm \sigma_{1}$ and $\pm \sigma_{2}$ are the strengths of monopoles, and $\pm \lambda_{1}$ and $\pm \lambda_{2}$ are the distance of each monopole to the origin respectively.

\subsection{Evolution in the System with Two-Dipole Boundary Condition}

In this part of work, we investigate the equilibrium and the evolution in the magnetic configuration with the background field produced by two dipoles located on the boundary surface (Figures 1a and 1b). The evolution in such a system is caused by the changes in either $\sigma$ or $\lambda$ or both. Plotting the equilibrium height of the flux rope $h$ versus $\lambda$ and $\sigma$ gives two equilibrium surfaces in $(\lambda, \sigma, h)$ space (Figure $2 \mathrm{a}$ ), which are in the regions of $\sigma>0$ and $\sigma<0$, respectively. The shadows located at the bottom of the figure outlines the domains of $(\lambda, \sigma)$ over which the equilibria in the system exists. Obviously, the two surfaces do not join one another within the domain of physical meaning $(\lambda>0$ and $h>0$ ). This implies that the smooth transition between the configurations in equilibria 

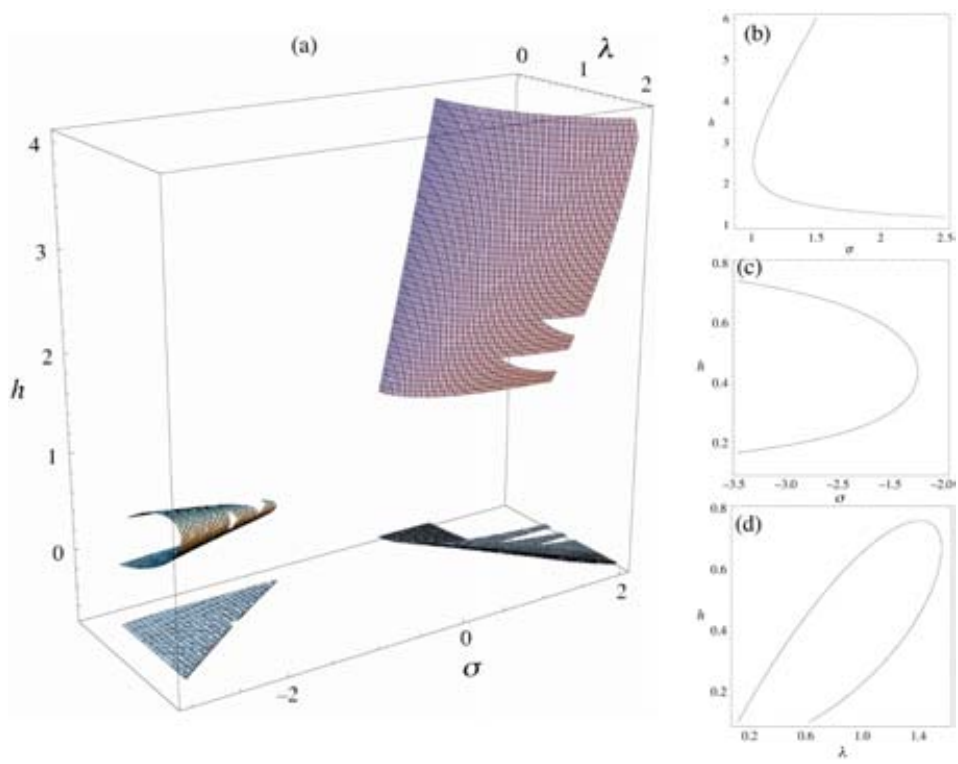

Figure 2. (a) Evolutionary features of the system manifested in the parameter space $(\lambda, \sigma, h)$. (b) Variations of the equilibrium heights $h$ versus $\sigma$ for $\lambda=1$ and $\sigma>0$. (c) Variations of $h$ versus $\sigma$ for $\lambda=1$ and $\sigma<0$. (d) Variations of $h$ versus $\lambda$ for $\sigma=-2.5$.

governed by different surfaces shown in Figure 2a is impossible since any such tendency will directly result in the catastrophic loss of equilibrium.

To reveal the detailed catastrophic behavior implied by Figure 2a, we slice the surfaces with a plane of $\lambda=$ const, say $\lambda=1$, and thereby obtain the functional behavior of $h$ as a function of $\sigma$. Two equilibrium curves ( $h$ versus $\sigma$ ) are deduced as shown in Figure 2b: the curve comes from the surface at right $(\sigma>0)$, and in Figure 2c: the curve comes from the surface at left $(\sigma<0)$. Both curves display the bifurcation and thus suggest the catastrophic characteristics of the evolution as the background field decays.

The evolution in the system may also occur in response to the change in $\lambda$, the distance between each source region and the origin. This equivalent to the case in which two active regions approach or depart from one another. Because we are just interested in the configuration with the flux rope located below the X-point (Figure 1a), we only need to focus on the evolution governed by the surface at left in Figure 2a. Slice this surface with a plane of $\sigma=-2.5$, thereby we obtain a curve of $h$ versus $\lambda$ as shown in Figure 2d. This curve consists of two branches and suggests the catastrophic behavior as the system evolves along the lower branch in response to the separation of two dipoles.

A similar situation with the point sources on the boundary surface was investigated by Forbes \& Priest (1995), but the quasi-static evolution in their case eventually turns to the catastrophe with the two source regions on the surface approaching, instead of separating from, one another. Furthermore, the range of $\lambda$ over which the equilibrium exists in the present case is much smaller than that of Forbes \& Priest (1995). Such differences in the evolutionary behaviors are basically due to the different properties of the background field in each case.

\subsection{Evolutions in the System with Boundary Condition of Four Monopoles}

In this part of work, we model the evolution in the system with the background field produced by four monopoles on the boundary surface. Comparing with the case of Forbes \& Priest (1995), two more monopoles are included. Adding two extra monopoles of 
opposite polarities to the magnetic system studied by Forbes \& Priest (1995) results in the magnetic configurations shown in Figure 1c and 1d. The complex boundary condition leads the X-point to existing in the background field. The interactions among different magnetic units in this system can be understood to occur either between the active region consisting of $-\sigma_{1}$ and $\sigma_{1}$ and two individual spots $-\sigma_{2}$ and $\sigma_{2}$, or between the two active regions of opposite orientations located at each side of the origin. For simplicity, we arrange these four monopoles in the symmetric way as shown in Figures 1c and 1d. In reality, the interacting systems may form much more sophisticated structures without any symmetry, but the fundamental physical courses should be the same.

Without losing the generality, we assume that $\lambda_{2}$ is always larger than $\lambda_{1}$, the evolution in the system is due to the change in either $\sigma_{1}$ or $\lambda_{1}$, and the sources $\pm \sigma_{2}$ exist as a background in distance. Such a scenario provides us with an opportunity to investigate how a large scale structure in background impacts the evolutionary behaviors of the local magnetic units, and how the re-organization of the local magnetic components in a confined area, in turn, disturbs the global field.

In this case, the equilibrium height of the flux rope $h$ is a function of $\sigma_{1}, \sigma_{2}, \lambda_{1}$, and $\lambda_{2}$. This is a five-dimensional function in $\left(\sigma_{1}, \lambda_{1}, \sigma_{2}, \lambda_{2}, h\right)$ space, which can hardly be investigated directly. In the present work, due to the limited space, we will focus on the evolutions in response to the changes in $\sigma_{1}$ and $\sigma_{2}$ only. Those in response to the changes in $\lambda_{1}$ and $\lambda_{2}$ can be found in our another work (Lin \& van Ballegooijen 2005). Figure 3a plots $h$ as a function of $\lambda_{1}$ and $\sigma_{1}$ for $\lambda_{2}=5$ and $\sigma_{2}=1$. (Gaps on the surface are purposely left so that the details behind the surface, if any, can be seen.) Figure $3 \mathrm{~b}$ displays a group of $h-\sigma_{1}$ curves for $\lambda_{1}=1,2,3$, and 4, respectively. All the curves show the typical property of bifurcation that results in the catastrophe as the system evolves in response to the change in the background field. Furthermore, the height of the turning (critical) point $\left(\sigma_{1 c}, h_{c}\right)$ at which the catastrophe occurs increases with the value of $\lambda_{1}$. This suggests that the eruption due to the magnetic cancellation between two approaching magnetic sources takes place more easily if the cancellation occurs as the two sources are still distant. We can understand this conclusion in another way: the equilibrium in the configuration with source regions close to one another can be kept more easily than that in the configuration of which the sources are located far apart.

Figure 3c plots $h$ versus $\lambda_{2}$ and $\sigma_{2}$ for $\lambda_{1}=\sigma_{1}=1$. We notice that when sources $\sigma_{1}$ and $\sigma_{2}$ are close to one another, the equilibrium occurs only if $\sigma_{2}<\sigma_{1}$. This is because strong $\sigma_{2}$ weakens the capability of $\sigma_{1}$ of maintaining the system in equilibrium. Figure $3 \mathrm{~d}$ plots the equilibrium curves, $h-\sigma_{2}$, for $\lambda_{2}=1.5,3,5$, and 10, respectively. These curves are nearly the duplicates of their counterparts in Figures $3 \mathrm{~b}$, except the opposite concave directions, which is indicating that the roles played by $\lambda_{1}$ and $\lambda_{2}$ (also by $\sigma_{1}$ and $\sigma_{2}$ ) in driving the evolutions of the system are just opposite. This suggests that the importance of sources $\sigma_{1}$ and $\sigma_{2}$ is relative, and their roles are symmetry in governing the evolution in the system.

\section{Conclusions}

Consequences of interactions between two magnetic systems (active regions) were investigated in the present work. The magnetic configurations include a current-carrying flux rope that is used to model the prominence or filament. The configurations in equilibrium evolve in response to the change in parameters for the background field, such as the strength and the distances between sources. Our main results are listed as follows:

1. The equilibrium positions of the flux rope can be either below or over the neutral points. In the case that the background field is produced by two dipoles, smooth transition 

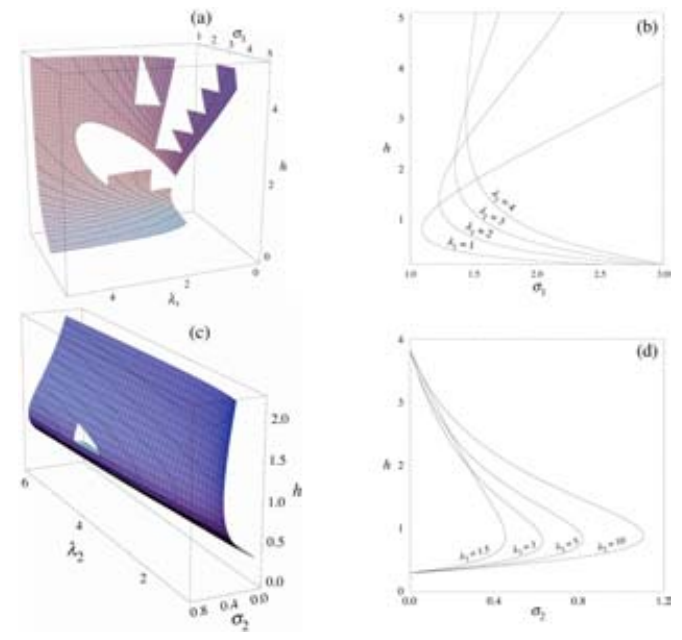

Figure 3. (a) Variations of the equilibrium heights of the flux rope $h$ versus $\lambda_{1}$ and $\sigma_{1}$. (b) Equilibrium curves $h-\sigma_{1}$ for various $\lambda_{1}$. (c) Variations of the equilibrium heights of the flux rope $h$ versus $\lambda_{2}$ and $\sigma_{2}$. (d) Equilibrium curves $h-\sigma_{2}$ for various $\lambda_{2}$.

between the configurations with the flux rope below and over the neutral points is impossible; in the case of four monopoles, such transition seems to be possible.

2. No matter whether the background field is produced by two dipoles or by four monopoles, evolutions of the system in response to the change in the sources may eventually perform catastrophic feature. This indicates the impact of the varying magnetic sources on driving the disruption of the magnetic structure in the corona.

3. We focus on the cases in which the flux rope is located below the neutral point. But the impact of the closed field lines over the neutral point on the consequence of the catastrophe is not clear. They may be quite possible to behave like those described by the break-out model (e.g., Antiochos et al. 1999; and MacNeice et al. 2004).

\section{Acknowledgements}

This work was supported by NASA under the grants NNG04GE84G and NAG5-12827 to the Smithsonian Astrophysical Observatory.

\section{References}

Antiochos, S.K., DeVore, C.R., \& Klimchuk, J.A. 1999, ApJ, 510, 485.

Dezsö, L., Csepura, G., Gerlei, O., Kovács, Á., \& Nagy, I. 1984, Adv. Space Rev., 4(7), 57.

Dezsö, L. \& Kovács, Á. 1998, in The 14th Consultation on Solar Physics: Conference Proceedings, (eds.) B. Rompolt, J. Jakimiec, \& P. Heinzel, p. 17.

Forbes, T.G. \& Priest, E.R. 1995, ApJ, 446, 377

Lin, J., Forbes, T.G., \& Isenberg, P.A. 2001, JGR, 106, 25053

Lin, J. \& van Ballegooijen, A.A. 2005, ApJ, submitted.

MacKay, D.H. \& van Ballegooijen, A.A. 2001, ApJ, 560, 445.

MacKay, D.H. \& van Ballegooijen, A.A. 2005, ApJ, 621, 77.

MacNeice, P.J., Antiochos, S.K., Phillips, A., Spicer, D.S., DeVore, C.R., \& Olson, K. 2004, ApJ, 614, 1028.

Martres, M.J., Mouradian, Z., \& Soru-Escaut, I. 1986, A\& A, 161, 376.

Tanaka, K. \& Nakagawa, Y. 1972, Solar Phys., 33, 187.

van Ballegooijen, A.A. \& Martens, P.C. H. 1989, ApJ, 343, 971.

van Ballegooijen, A.A., Priest, E.R. \& MacKay, D.H. 2000, ApJ, 539, 983. 


\section{Discussion}

P. F. Chen: Forbes \& Priest (1995), compared two solutions at the critical point, one with a current sheet, and the other without a current sheet. Have you considered this in your work?

LIN: In fact, the solution deduced by Forbes \& Priest (1995) consists of two components, one for the configuration including a vertical current sheet attached to the boundary surface and another one for the without the current sheet. With the specific background field that they chose, the current sheet did not develop below the flux rope in the relevant magnetic field until the system loses the mechanical equilibrium. In the present case, on the other hand, the magnetic configuration possesses more complex structure since the extra magnetic sources are included. The complexity of such structure can be seen first from the X-type neutral point or the vertical current sheet of finite length located above the current sheet prior to the eruption, and further from the multiple patterns of the catastrophe shown by the evolutionary behaviors of the system in response to the change in the background field. In our work, we considered the situation in which a vertical current sheet of finite length is located above the flux rope during the slow evolution. But whether such a configuration makes any sense in reality is not clear. For the time being, we could not find the closed form of the solution for the evolution in the magnetic configuration that includes a vertical current sheet attached to the boundary surface due to the complexity in mathematics. Further investigation on this issue is worthwhile and will be arranged for our work in the future. 